\title{
AVALIAÇÃO DA QUALIDADE DO ESCARRO OBTIDO ATRAVÉS DA UTILIZAÇÃO DE TÉCNICAS FISIOTERAPÊUTICAS EM PACIENTES COM DPOC ESTÁVEL
}

Sputum quality evaluation obtained by the use of physical therapy techniques in patients with stable copd

Ana Lucia Bernardo de Carvalho Morsch ${ }^{1}$; Mariluce da Rocha Jaskulski ${ }^{2}$; Dechristian França Barbieri ${ }^{3}$; Felipe Knack Osório ${ }^{4}$; Fernanda Fiabanne ${ }^{4}$; Deomar Pawlak ${ }^{5}$

${ }^{1}$ Professora do Curso de Fisioterapia da Universidade Regional Integrada do Alto Uruguai e das Missões

- URI Erechim, Doutora em Ciências da Saúde pela UNESC - Criciúma.

${ }^{2}$ Professora do Curso de Farmácia da Universidade Regional Integrada do Alto Uruguai e das Missões - URI Erechim, Doutora em Medicina e Ciências da Saúde pela PUC/RS.

${ }^{3}$ Pós-doutorando em Fisioterapia pela Universidade Federal de São Carlos (UFSCar), São Carlos, SP.

${ }^{4}$ Fisioterapeuta Diplomado pela Universidade Regional Integrada do Alto Uruguai e das Missões - URI Erechim.

${ }^{5}$ Farmacêutico Graduado pela Universidade Regional Integrada do Alto Uruguai e das Missões - URI Erechim.

RESUMO: A coleta de escarro é um procedimento de simples execução, muito solicitado para pacientes com DPOC (Doença Pulmonar Obstrutiva Crônica), utilizado para avaliar a presença de inflamação nas vias aéreas, porém com alto índice de contaminação devido à realização incorreta da coleta. O objetivo do estudo foi analisar a influência de duas técnicas fisioterapêuticas na qualidade analítica do escarro. A amostra foi composta por 13 indivíduos com diagnóstico de DPOC, divididos em dois grupos: Grupo A - Oscilação oral de alta frequência $(n=6)$ com indução de escarro pelo recurso Shaker ${ }^{\circ}$; Grupo B - Técnica de expiração forçada (TEF): $(n=7)$ com indução de escarro pela técnica Huffing. Os indivíduos foram submetidos a espirometria, a manovacuometria e responderam ao escore de tosse diurna e noturna. A amostra foi coletada por meio da técnica pré-definida sendo, posteriormente, encaminhada 
para análise em laboratório. Verificou-se que as técnicas foram igualmente seguras e eficazes na indução de escarro, com uma boa qualidade analítica, porém, devido ao grupo amostral pequeno, não foi possível observar diferença estatisticamente significativa. Não houve correlação entre as variáveis PRM, $\mathrm{VEF}_{1}$ e qualidade da amostra com o escore de tosse.

Palavras-chave: DPOC. Escarro Induzido. Fisioterapia.

\begin{abstract}
Sputum collection is a simple procedure that is often indicated for COPD patients to evaluate airway inflammation, but with a high rate of contamination due to incorrect procedure. The aim of the present study was to analyze the influence of two physiotherapeutic techniques on the sputum analytical quality. The sample consisted of 13 subjects diagnosed with COPD, divided into two groups: group A- High frequency oral oscillation: $(n=6)$ sputum induction with Shaker ${ }^{\circledR}$ resource; group B- Forced Exhalation Technique (FET): $(n=7)$ sputum induction with the Huffing technique. The subjects underwent spirometry, manovacuometry and responded to the day and night cough score. The sample was collected by the predefined technique and then sent for laboratory analysis. It was found that the techniques were equally safe and effective in sputum induction to a good analytical quality, but due to the small sample group it was not possible to observe a statistically significant difference. There was no correlation between the PRM, FEV and sample quality variables with the cough score.
\end{abstract}

Keywords: COPD. Induced Sputum. Physiotherapy.

\section{Introdução}

A doença pulmonar obstrutiva crônica (DPOC) é uma das principais causas de morbidade e mortalidade em todo o mundo e pode-se atribuir grande parte dos casos ao tabagismo (VLAHOS; BOZINOVSKI, 2014). No Brasil, 14.320 milhões de indivíduos têm DPOC relacionada ao tabagismo, sendo a quinta causa de maior mortalidade no país e responsável por 3 milhões de mortes a cada ano no mundo (RABAHI, 2013; AZAMBUJA, 2013).

A DPOC caracteriza-se pela limitação do fluxo aéreo associado a uma exacerbada resposta inflamatória nos pulmões relacionada a gases e partículas nocivas. Há evidências de fenótipos diferentes da doença como fibrose e obstrução de pequenas vias aéreas, enfisema com alargamento dos espaços aéreos e destruição do parênquima pulmonar, perda do recuo elástico e obstrução das vias aéreas de pequeno calibre (TUDER; PETRACHE, 2012). A severidade da doença é atualmente classificada de acordo com o projeto GOLD - Iniciativa Global para a Doença Pulmonar Obstrutiva Crônica (do inglês: Global Initiative on Chronic Lung Disease) nos critérios de uma escala de 0-4 baseada na deterioração da função pulmonar e sintomas (GOLD, 2018).

Uma das mais importantes manifestações clínicas do paciente com DPOC é um aumento da produção de secreção das vias aéreas, o 
que facilita a colonização bacteriana das vias aéreas. $\mathrm{O}$ exame de escarro costuma ser o mais utilizado para detectar microrganismos na secreção, que pode ser constituído de material de natureza diversa, ampla, representando áreas pulmonares só acessíveis aos métodos invasivos. A análise do escarro pode contribuir de forma decisiva para o diagnóstico de um grande número de doenças do aparelho respiratório, como pneumonia, asma, DPOC e turberculose (TARANTINO, 2002; BRIGHTLING, 2006).

Segundo Brightling (2006), a utilização da análise do escarro como um instrumento de investigação foi inicialmente dificultada por uma falta de padronização e, em muitos casos, da incapacidade de obter uma amostra. Muitas vezes o exame tem de ser repetido, aumentando efetivamente os gastos. $\mathrm{O}$ objetivo principal ao se analisar uma amostra de escarro é obtê-la da forma mais adequada possível, independente de qual método for escolhido para sua obtenção. Considera-se uma boa amostra de escarro aquela com volume superior a $3 \mathrm{ml}$, cor verde-amarelado (purulento), com aparência viscosa (Sakundarno 2009), com menos de 10 células epiteliais/ campo e mais que 25 leucócitos/campo (MÖLLER et al., 2002).

Atualmente, a coleta do escarro pode ser feita de diferentes formas, considerando a condição do paciente e a conduta de escolha do profissional que solicita o exame. Comumente, opta-se pela coleta simples, em que o paciente é orientado a coletar o escarro pela manhã, após lavar a boca com água, respirar fundo e tossir profundamente, produzindo uma amostra de escarro que deve ser armanezado em recipiente estéril. Nos casos em que o paciente apresenta dificuldade para expectorar espontaneamente, pode-se realizar a indução do escarro com solução salina. Em alguns casos, o médico pode optar por realizar a coleta por meio de broncoscopia (DATTA, 2017; KENNEDY,
1999). Embora a assistência da fisioterapia no auxílio de obtenção de amostra de escarro tenha sido pouco documentada, o estudo de LANGRIDGE (2016) mostra que o emprego de treinamento respiratório pode auxiliar na obtenção de melhor amostra.

De acordo com um estudo realizado por Morsch et al. (2008), a utilização de técnicas de fisioterapia respiratória em um protocolo padrão de indução de escarro com solução salina influenciou o resultado das amostras de escarro coletadas em pacientes estáveis, como o aumento do peso do escarro em pacientes com asma, assim como aumento do número absoluto de células em pacientes com asma e DPOC, influenciando na qualidade do mesmo. Contudo, não é sabido se o emprego dessas técnicas em um protocolo de coleta de escarro simples (não induzido através de solução hipertônica) levaria a um aumento da quantidade e melhor qualidade de material expectorado. Assim sendo, o presente estudo teve, como objetivo central, analisar se o emprego das técnicas fisioterapêuticas de oscilação oral de alta frequência e de expiração forçada são efetivas na coleta de escarro com boa qualidade analítica. Secundariamente, investigou-se se uma técnica é superior a outra, quando correlacionados a pressão respiratória máxima (PRM), o volume forçado no primeiro segundo $\left(\mathrm{VEF}_{1}\right)$ e o escore de tosse com a qualidade de escarro.

\section{Material e Métodos}

O presente estudo foi do tipo observacional de corte transversal, de cunho exploratório, descritivo e de caráter quantitativo. $\mathrm{O}$ estudo se caracteriza também por ser simples-cego, uma vez que só o fisioterapeuta sabia em qual grupo o paciente foi alocado após aleatorização. A análise das amostras também foi realizada de forma cega, uma vez que o encarregador pelas análises não conhecia os 
grupos e técnicas empregadas. O estudo foi aprovado pelo Comitê de Ética em Pesquisa em Seres Humanos sob número 061/PPH/09.

A amostra foi composta por 13 pacientes, de ambos os sexos, residentes no município de Erechim, RS, encaminhados pelos médicos pneumologistas da cidade com diagnóstico de DPOC $\left(\mathrm{VEF}_{1} / \mathrm{CVF}<0,70 ; \mathrm{VEF}_{1} \geq\right.$ $80 \%$ do previsto), moderado $\left(\mathrm{VEF}_{1} / \mathrm{CVF}<\right.$ 0,$70 ; 50 \% \leq \mathrm{VEF}_{1}<80 \%$ do previsto) a grave $\left(\mathrm{VEF}_{1} / \mathrm{CVF}<0,70 ; 30 \% \leq \mathrm{VEF}_{1}<50 \%\right.$ do previsto), segundo classificação de GOLD (2016), com nível cognitivo que permitia o entendimento para realização dos testes propostos e que manifestassem interesse em participar do estudo. Foram excluídos os indivíduos que não conseguiram executar as manobras dos testes propostos ou que não concordaram em assinar o Termo de Consentimento Livre e Esclarecido (TCLE).

Para avaliação da função pulmonar, primeiramente os pacientes foram submetidos a um teste espirométrico com aparelho Micromedical, modelo Spirolab II, de acordo com a metodologia descrita por PEREIRA (2001) e para obtenção das pressões respiratórias máximas (PRM), a manovacuometria foi realizada por meio do manovacuômetro digital modelo MVD 300 da marca Globalmed, seguindo a metodologia de Neder (1999).

Após a realização dos testes, os pacientes foram randomizados através de sorteio simples e submetidos a uma das duas técnicas fisioterapêuticas com data marcada para ser realizada (pois os testes de função pulmonar poderiam afetar o resultado do escarro). Em relação aos grupos, o grupo A Shaker foi submetido a técnica de oscilação oral de alta frequência (OOAF) com o recurso de Shaker ${ }^{\circledR}$; grupo B TEF (7 pacientes): indução de escarro com a técnica de expiração forçada (TEF). O componente responsável pela análise das amostras de escarro foi cego, não sabendo de qual grupo a amostra pertencia.
No momento da coleta, os pacientes foram orientados pelo pesquisador a realizar higiene oral que consistia em três bochechos de água destilada e após a higiene nasal, além da ingestão de água, para posteriormente iniciar a indução e coleta de escarro. O material coletado foi armazenado no interior de potes de polipropileno (estéreis), que foram fechados e rotulados com um código numeral, de forma que o avaliador que realizou a análise das lâminas não sabia a que grupo pertencia o material, logo após sendo encaminhado para análise. O início deste procedimento não ultrapassou o período de duas horas, como recomendado por alguns autores, para não comprometer o material colhido (MENDES, 2005; OPLUSTIL, 2004). Os pacientes do grupo A Shaker foram instruídos a sentar em posição ereta e realizar expirações tranquilas, porém longas, com o aparelho Shaker ${ }^{\circledR}$, durante 5 minutos ininterruptos, com o mesmo na posição horizontal. Depois foi solicitado ao paciente uma tosse vigorosa, seguida de expectoração em recipiente de coleta descartável. Os pacientes do grupo B TEF foram instruídos a sentar em posição ereta e realizar a técnica de expiração forçada (TEF) que consistiu em uma inspiração profunda, e em seguida com a glote aberta contraiu a musculatura abdominal e realizou uma expiração forçada. A TEF foi realizada 10 vezes, em intervalos de $30 \mathrm{~s}$, seguidas de tosse vigorosa e expectoração. Nos dois grupos o procedimento foi interrompido quando a quantidade de escarro era suficiente para a análise (100 miligramas), quando apresentaram sinais de desconforto e/ou cansaço ou quando o paciente solicitou para interromper a coleta. As amostras foram enviadas ao laboratório de microbiologia em temperatura ambiente, em um prazo máximo de até 2 horas após a coleta e, para períodos maiores, foram refrigeradas de $2-8^{\circ} \mathrm{C}$ sendo analisadas dentro de um período máximo de 12 horas. Elas não eram identificadas sobre 
qual método de coleta foram originadas, sendo o analisador do escarro cego em relação ao procedimento. Para análise macroscópica do escarro, este foi retirado do recipiente e após colocado em uma placa de Petri para uma melhor visualização e detecção do que era escarro ou saliva. Desta forma pôde-se fazer uma análise macroscópica da amostra de acordo com sua consistência. Para a análise microscópica de cada amostra foi feito um esfregaço fino em lâmina, selecionando-se a parte mais purulenta com auxílio de uma alça bacteriológica. Em seguida foi realizada a coloração de Gram. Após esta etapa, a lâmina foi levada ao microscópio, onde se realizou uma minuciosa análise e as mesmas foram observadas com objetiva de 10X (aumento de 100X) pelo menos 10 campos microscópicos. Posteriormente foi realizado o critério de qualificação da amostra verificando-se a média de leucócitos e células epiteliais por campo. $\mathrm{Na}$ avaliação microscópica foi considerada uma amostra de boa qualidade ou adequada aquela que apresentou $\leq 10$ células epiteliais/ campo e $\geq 25$ leucócitos/campo. As amostras contendo mais do que 10 células epiteliais/ campo e menos que 25 leucócitos, foram consideradas de baixa qualidade ou inadequadas. Quando o escarro não apresentava as características descritas anteriormente, relatou-se que o quadro era consistente com contaminação de orofaringe (amostra não representativa, processamento não indicado). Nesta mesma lâmina foi realizada uma análise em maior aumento (1000x) com a objetiva de imersão segundo as características morfológicas do Gram relatando-se os eventuais microrganismos presentes na amostra. Foi confeccionada uma segunda lâmina com um esfregaço fino, selecionando-se a parte mais purulenta com auxílio de uma alça bacteriológica realizando a coloração Instant Prov ${ }^{\circledR}$ analisando-se, nesta lâmina, o citológico diferencial em aumento de (1000x) com a objetiva de imersão, observando-se o percen- tual de neutrófilos, macrófagos, eosinófilos e linfócitos. Esta diferença de celularidade é de grande importância para o clínico dar assistência ao seu paciente, assim como também é relevante o número de neutrófilos, macrófagos que na DPOC se encontram elevados e os eosinófilos que poderão estar associados a reações a corticosteroides. Estes resultados obtidos foram registrados na ficha de análise laboratorial de escarro.

\section{Análise Estatística}

Para tratamento dos dados, as variáveis coletadas foram tabuladas e arquivadas em planilhas do programa Microsoft Excel 2000 e após foram analisadas no programa estatístico Bioestat 5.0. Foi realizada uma análise descritiva das variáveis independentes e as correlações entre as variáveis dependentes do estudo utilizando o coeficiente de correlação linear simples de Pearson e o teste Mann-Whitey para comparar as variáveis de ambos os grupos.

\section{Resultados}

A população foi composta inicialmente por 20 participantes, sendo excluídos três pacientes por não conseguirem executar as manobras dos testes propostos e quatro pacientes por não apresentarem produção de escarro. Sendo assim, a amostra foi composta por 13 voluntários, seis sujeitos do grupo $\mathrm{A}$ e sete sujeitos do grupo B. As características das amostras são apresentadas na Tabela I. As variáveis clínicas (Tabela I) foram testadas entre os grupos, as quais não demonstraram diferença significativa $\left(\mathrm{VEF}_{1}, \mathrm{~L} \%, \mathrm{p}=0,39\right.$; Pimáx, $p=0,20$ e PEmáx, $p=0,89$ ). 
Tabela I - Caracterização da amostra

\begin{tabular}{lll}
\multicolumn{1}{c}{ Características } & \multicolumn{1}{c}{ A Shaker } & B TEF \\
\hline Gênero M/F, $\mathrm{n}^{\circ}$ & $4 / 2$ & $5 / 2$ \\
Idade, anos (média/DP) & $67 \pm 6,93$ & $66,29 \pm 6,16$ \\
Variáveis clínicas (média/DP) & & \\
$\mathrm{VEF}_{1}, \mathrm{~L} \%$ & $1,57 \pm 0,75$ & $1,34 \pm 0,53$ \\
Pimáx & $68,50 \pm 25,59$ & $96,71 \pm 46,09$ \\
PEmáx & $109,50 \pm 29,56$ & $126,57 \pm 55,72$ \\
Gravidade da DPOC, $\mathrm{n}^{\circ}$ & & \\
Leve & 1 & 0 \\
Moderado & 3 & 2 \\
Grave & 2 & 5 \\
\hline
\end{tabular}

Valores expressos em média e desvio padrão; $\mathrm{M}$ : masculino; $\mathrm{F}$ : feminino; $\mathrm{VEF}_{1}$ : volume expiratório forçado no primeiro segundo; TEF: técnica de expiração forçada; PImax: pressão Inspiratória máxima; PEmax: pressão expiratória máxima

A Tabela II apresenta a divisão dos grupos de acordo com a análise macroscópica das amostras em aquosa, mucoide e mucopurulenta.

Tabela II - Classificação das amostras segundo as análises macroscópicas

\begin{tabular}{l|l|l|l|l}
\hline \multirow{2}{*}{ Análise Macroscópica } & \multicolumn{2}{|c|}{ A Shaker } & \multicolumn{2}{c}{ B TEF } \\
\cline { 2 - 5 } & $\mathrm{n}$ & $\%$ & $\mathrm{n}$ & 14 \\
\hline Aquosa & 2 & 33 & 1 & 86 \\
Mucoide & 4 & 67 & 6 & 0 \\
Mucopurulenta & 0 & 0 & 0 & 0 \\
\hline
\end{tabular}

TEF: técnica de expiração forçada

Para a qualidade do escarro obtido nas amostras foi realizada a análise microscópica por meio da contagem das células epiteliais/ campo e dos leucócitos/campo. Conforme dados apresentados na Tabela III, a média da variável célula epiteliais/campo para o grupo
$\mathrm{B}$ foi maior que o grupo A, porém não houve diferença estatística entre os grupos $(\mathrm{p}>0,05)$. O grupo B apresentou três amostras classificadas como inadequadas, enquanto o grupo A apresentou duas amostras inadequadas.

Tabela III - Qualidade do escarro

\begin{tabular}{llll}
\hline Análise Microscópica & A Shaker* & B TEF* & p \\
\hline Leucócitos/campo & $87,33 \pm 85,26$ & $66,99 \pm 98,52$ & 0,568 \\
Células epiteliais/campo & $9,62 \pm 35,30$ & $14,51 \pm 14,11$ & 0,668 \\
\hline
\end{tabular}

*Os valores estão expressos em média e desvio padrão; $<<0,05$; TEF: técnica de expiração forçada 
Ao correlacionar as pressões respiratórias máximas, volume expiratório forçado no primeiro segundo, escore de tosse e qualida- de da amostra em ambos os grupos, não se observou correlação dos parâmetros entre si (Tabela IV).

Tabela IV - Correlação das variáveis para o grupo A Shaker e B TEF

\begin{tabular}{llll}
\hline Variável & & A Shaker & B TEF \\
\hline 1 - PI max & VEF $_{1}$ & Fraca $(\mathrm{r}=0,23)$ & Fraca $(\mathrm{r}=0,17)$ \\
2 - PE max & VEF $_{1}$ & Fraca $(\mathrm{r}=0,30)$ & Regular $(\mathrm{r}=0,42)$ \\
3 - Tossediu & Células epit & Fraca $(\mathrm{r}=0,09)$ & Regular/Forte $(\mathrm{r}=0,59)$ \\
$4-$ Tossediu & Leucócitos & Regular $(\mathrm{r}=0,43)$ & Fraca $(\mathrm{r}=0,28)$ \\
$5-$ Tossenot & Células epit & Fraca $(\mathrm{r}=0,27)$ & Forte $(\mathrm{r}=0,62)$ \\
6 - Tossenot & Leucócitos & Fraca $(\mathrm{r}=0,07)$ & Regular $(\mathrm{r}=0,47)$ \\
7 - Qualidade & Tossediu & Fraca $(\mathrm{r}=0,21)$ & Regular $(\mathrm{r}=0,47)$ \\
$8-$ Qualidade & Tossenot & Inexistente $(\mathrm{r}=0,00)$ & Forte $(\mathrm{r}=0,62)$ \\
\hline
\end{tabular}

Coeficiente de correlação de Pearson. r: índice de correlação; TEF: técnica de expiração forçada; PImax: pressão inspiratória máxima; PEmax: pressão expiratória máxima; $\mathrm{VEF}_{1}$ : volume expiratório forçado no primeiro segundo; Tossediu: tosse diurna; Tossenot: tosse noturna; Células epit: células epiteliais; Qualidade: qualidade da amostra.

Os valores de PImáx, PEmáx, $\mathrm{VEF}_{1}$, escore de tosse diurna e escore de tosse noturna foram comparados entre os grupos por meio do teste estatístico Mann-Whitney, o qual não detectou nenhuma diferença significativa para as variáveis ( $\mathrm{p}>0.005)$.

\section{Discussão}

Como principal achado deste estudo, observou-se em pacientes com DPOC, de leve a grave, que as técnicas de oscilação oral de alta frequência e de expiração forçada foram efetivas na indução de escarro com boa qualidade analítica.

No estudo realizado por Morsch et al. (2008), 100\% dos pacientes com DPOC apresentaram sucesso na coleta de escarro para a análise, enquanto que apenas $75 \%$ dos pacientes asmáticos apresentaram escarro suficiente para a análise, porém a técnica utilizada no protocolo de indução foi com solução salina hipertônica (o que favoreceu a eliminação do escarro). No atual estudo, 7 amostras foram excluídas devido à dificuldade do paciente de realizar a técnica ou por não apresentarem a produção de escarro. A técnica de indução utilizada no presente estudo foi padrão, ou seja, sem utilização de soluções para auxiliar na indução do escarro. Pode-se inferir que, nesta população, o emprego das técnicas e recursos fisioterapêuticos não foram eficazes para deslocar secreções das vias aéreas mais distais para as mais centrais. Ademais, os indivíduos portadores de DPOC têm fenótipos diferentes, sendo que uns produzem maior quantidade de secreção (bronquíticos) e outros apresentam como característica principal a dispneia devido à hiperinsuflação pulmonar (enfisematosos), o que pode contribuir com a exaustão durante o procedimento de coleta levando à interrupção das manobras. No entanto, a confirmação do fenótipo é feita a partir de exames mais complexos e que muitas vezes não são acessíveis para uma parte da população. Há muitos anos se discute a heterogeneidade da doença, e 
futuramente a identificação de fenótipos de DPOC na prática clínica seria interessante principalmente objetivando uma terapia individualizada (BÁRTHOLO; COSTA, 2017).

Das três amostras aquosas, sendo duas do grupo A Shaker, uma do grupo B TEF e duas amostras mucoides do grupo B TEF constituíram as cinco amostras consideradas inadequadas ou de baixa qualidade, corroborando com os resultados apresentados por Murray e cols. (1975 apud VALENÇA, 1989). Tais autores mostraram que o aspecto macroscópico do escarro e a presença de leucócitos e macrófagos em números variados não são dados confiáveis para indicação de contaminação orofaríngea. Outro fator que pode interferir na consistência, na quantidade e qualidade do escarro expectorado para análise é o período em que é coletado, sendo que o escarro coletado no período matutino tende a ser mais adequado devido ao acúmulo de secreção que tende a ser maior no período noturno e com isso o aumento de células inflamatórias em vias aéreas inferiores (RUFINO, 2007; SILVA, 2004). Além disso, uma amostra de boa qualidade é composta por secreções da árvore brônquica (células inflamatórias), sendo inadequadas amostras provenientes da faringe, amostras obtidas por aspiração de secreções nasais, ou ainda que contêm somente saliva em sua composição, sendo considerado de aspecto ideal uma amostra de escarro mucopurulento (LOSCALZO, 2014). Uma justificativa para a condição das amostras observadas no presente estudo é o fato de que os pacientes com DPOC estavam estáveis, ou seja, provavelmente sem infecção das vias aéreas, resultando em um escarro inadequado para análise. Uma limitação do presente estudo foi a não padronização do período de coleta das amostras, pois dependia da disponibilidade e rotina do paciente.

Falqueto et al. (2006), em seu estudo, alternaram as coletas de amostra de escarro com e sem orientação, sempre no período matutino após o desjejum. A orientação, quando realizada, seguia as normas preconizadas pelo Ministério da Saúde, tendo como parâmetro avaliativo a análise microscópica, classificando o material quanto à representatividade ou não das vias aéreas inferiores e os dois grupos se deram homogêneos quanto aos parâmetros avaliados. No entanto, não houve diferença estatisticamente significante entre as amostras com e sem orientação. Alguns autores relatam que a utilização de métodos para diminuir a contaminação, como lavagem bucal e bochecho, apenas com água, ou secagem da boca, podem aumentar o nível de contaminação da região orofaríngea (PAGGIARO et al., 2002). No presente estudo foi realizado um protocolo de higienização das vias aéreas, o que pode ter contribuído para a característica das amostras.

Pode-se observar que o grupo B TEF apresentou uma maior média de PImáx e PEmáx quando comparado ao grupo A Shaker (Tabela I), porém esta diferença não foi estatisticamente significativa. Ao analisarmos as variáveis 1 e 2, ou seja, a correlação entre PImáx e VEF ${ }_{1}$ e PEmáx e VEF ${ }_{1}$, observou-se uma fraca correlação em ambos os grupos, sendo regular para PEmáx e $\mathrm{VEF}_{1}$ no grupo B TEF, vindo ao encontro dos estudos de Lopes et al. (2009), o qual avaliou 35 indivíduos portadores de DPOC com idade média de 65,88 \pm 9,52 anos e correlacionando as PRM com $\mathrm{VEF}_{1}$ por meio do teste de correlação de Pearson, o mesmo não encontrou correlação significativa entre o $\mathrm{VEF}_{1}$ e a PImáx e PEmáx. Sipoli et al. (2009), em seu estudo avaliando 53 pacientes portadores de DPOC, encontraram fraca correlação entre PImáx e $\mathrm{VEF}_{1}$, sendo esta estatisticamente significativa. De acordo com o presente estudo, as médias obtidas de PImáx e PEmáx foram mais altas no grupo B TEF quando comparadas ao grupo A Shaker, de acordo com a Tabela 4, não sendo estatisticamente significativas. Entretanto, a hipótese de que o 
grupo B TEF apresentou maiores PRM pelo fato de o grupo ser composto por pacientes com diagnóstico de DPOC menos graves que o grupo A Shaker não se confirmou, pois o grupo B TEF foi constituído de cinco pacientes graves e dois moderados, enquanto o grupo A Shaker se constituiu de um paciente leve, três moderados e dois graves, de acordo com os resultados da Tabela 4, em que a média do $\mathrm{VEF}_{1}$ foi maior para o grupo $\mathrm{A}$ Shaker $(1,57 \mathrm{~L})$ do que para o grupo B TEF $(1,34 \mathrm{~L})$.

$\mathrm{O}$ grupo B TEF apresentou maior índice de correlação quando comparado ao grupo $\mathrm{A}$ Shaker nas variáveis $2,3,5$ e 6 . No entanto, estes resultados podem estar relacionados com um número maior da amostra do grupo B TEF e uma média de células epiteliais/ campo maior e uma média de leucócitos/ campo menor que o grupo A Shaker, conforme Tabela III.

Foi encontrada correlação entre a qualidade do escarro e escore da tosse (variáveis 7 e 8) apenas para o grupo B-TEF, sendo regular para tosse diurna e forte para tosse noturna. Podendo-se relacionar com o fato de o grupo B TEF ser composto por pacientes com diagnóstico de DPOC mais grave que os do grupo A Shaker. Somando, assim, o diagnóstico mais grave com a diminuição normal e gradual do $\mathrm{VEF}_{1}$ pelo envelhecimento, pode-se determinar maior obstrução das vias aéreas, produzindo, conseguintemente, escarros de melhor qualidade analítica. No entanto, não foram encontradas literaturas que analisassem a correlação de tais variáveis. De acordo com o estudo realizado por Morsch et al. (2008), o escore de tosse diurna e noturna foi menor nos pacientes portadores de asma do que nos portadores de DPOC (valor da mediana, 1 e 2, respectivamente; $p<0,01)$. No atual estudo, $o$ grupo estudado foi homogêneo, caracterizado por pacientes portadores de DPOC. Parr et al. (2006) demonstrou, em seu estudo, que níveis mais elevados de vários marcadores inflamatórios no escarro estão associados com uma maior taxa de declínio subsequente na função pulmonar e, além disso, que o nível de escarro IL-8 está associado com posterior progressão do enfisema. Em outro estudo, realizado por Bizeto et al. (2008), os marcadores séricos inflamatórios não se correlacionaram com o grau de comprometimento da função pulmonar no grupo de pacientes com DPOC estável.

Não foi encontrada diferença estatisticamente significante entre os grupos A Shaker e B TEF, demonstrando que ambas as técnicas são igualmente seguras para a indução de escarro nos pacientes com DPOC, no entanto os tempos de intervenção necessária em cada grupo para a indução do escarro não foi observado. A não significância dos resultados possivelmente ocorreu devido ao número de amostra pequeno em ambos os grupos, e de acordo com a Tabela 4, apenas oito amostras foram classificadas como adequadas ou de boa qualidade, sendo quatro amostras de cada grupo. Tal resultado pode estar relacionado com a composição heterogênea da amostra, sendo os grupos compostos por DPOC leve, moderado e grave.

Bellone et al. (2000) compararam os efeitos da drenagem postural, oscilação oral de alta frequência (OOAF) e expiração lenta com a glote aberta em decúbito lateral em pacientes com bronquite crônica. Os autores observaram um aumento da produção de escarro, obtido após 30 minutos de intervenção, após a aplicação de todas as técnicas de fisioterapia, sendo que o aumento mais significante da produção de escarro ocorreu quando utilizada a técnica OOAF. No estudo realizado por Morsch et al. (2008), os pacientes com DPOC apresentaram menor tempo (20 minutos) para a obtenção do escarro após as intervenções em relação ao tempo observado para os indivíduos asmáticos.

Uma das limitações do presente estudo, além da amostra pequena, foi a ausência da estipulação de um tempo para a obtenção da coleta em ambos os grupos. Sugerem-se futuras pesquisas com essa abrangente temática. 


\section{Considerações Finais}

Conclui-se com este estudo que o emprego das técnicas de oscilação oral de alta frequência e de expiração forçada são efetivos na coleta de escarro com boa qualidade analítica, sem diferença entre elas. Não houve correlação entre as variáveis PRM, $\mathrm{VEF}_{1}$, qualidade da amostra e escore de tosse.
Destaca-se o fato de alguns sujeitos do estudo não terem conseguido realizar as técnicas propostas. Diante disso, sugerem-se pesquisas com maior amostra, que visem a compreender esse achado. Ainda, futuros estudos podem investigar a efetividade de outras técnicas fisioterapêuticas que auxiliem na coleta de escarro em grupos de DPOC de acordo com a gravidade da doença.

\section{REFERÊNCIAS}

AZAMBUJA, R. et al. Panorama da doença pulmonar obstrutiva crônica. Revista HUPE, v. 12, n. 2, p.13-18, 2013.

BÁRTHOLO TP; COSTA CH. Fenótipos da DPOC: há interesse prático nesta avaliação? Pulmão, v. 26, n. 1, p. 23-28, 2017.

BELLONE, A.; LASCIOLI, R.; RASCHI, S.; GUZZI, L.; ADONE, R. Chest physical therapy in patients with an acute exacerbation of chronic bronchitis: effectiveness of three methods. Arch Phys Med Rehabil, v. 81, n. 5, p.558-560, 2000.

BIZETO, L. et al. Interrelationship between serum and sputum inflammatory mediators in chronic obstructive pulmonary disease. Brazilian Journal of Medical and Biological Research, v. 41, n. 3, p.193-198, 2008.

BRIGHTLING, E. C. Clinical Applications of Induced Sputum. Chest, v.129, n.5, p. 1344-1348, 2006.

BRIGHTLING, E. C. Sputum Induction in Asthma. Chest, v. 129, n.3, p. 503-504, 2006.

DATTA, S., SHAH L., GILMAN R. H., EVANS, A. C. Comparison of sputum collection methods for tuberculosis diagnosis: a systematic review and pairwise and network meta-analysis. Lancet Glob Health, v.5, n. 8, e760-e771, 2017.

FALQUETO, L. et al. Impacto da orientação na coleta do escarro sobre a qualidade da amostra obtida. Arquivos Catarinenses de Medicina, v. 35, n. 3, p. 29-34, 2006.

GOLD. Global strategy for Diagnosis, Management, and Prevention of COPD, 2016.

KENNEDY, T.C. et al. Efficacy of Two Sputum Collection Techniques in Patients with Air Flow Obstruction. Acta Cytologica, v. 44, n. 4, p. 630-6, 1999.

LANGRIDGE, P.J., SHEEHAN, R.L., DENNING, D.W. Microbial yield from physioterapy assisted sputum production in respiratory outpatients. BMC Pulmonary Medicine, v. 16, n. 23, p. 1-8, 2016.

MADHAVI, S. RAMA, R.M.V. JANARDHAN, R. R. Bacterial etiology of acute chronic obstructive pulmonar disease. Journal of Microbiology and Biotechnology Research, v. 2, n. 3, p.440 - 444, 2013.

LOPES, V. S. et al. Estudo da relação entre o $\mathrm{VEF}_{1}$ e as pressões respiratórias máximas em indivíduos com DPOC. Revista Inspirar, v. 1, n. 3, p. 84, 2009. 
MANNINO, D. M. AND A. S. BUIST. Global burden of COPD: risk factors, prevalence, and future trends. Lancet, v. 370, n. 9589, p. 765-773, 2007.

MENDES, C.M.F.et al. Microbiologia Clínica -156 perguntas e respostas. São Paulo: Sarvier, 2005. MÖLLER, J. L. et al. Métodos diagnósticos, consulta rápida. 1. ed. Porto Alegre: Artmed, 2002. MORSCH, A.L.B.C. et al. Influência da técnica de pressão expiratória positiva oscilante e da técnica de expiração forçada na contagem de células e quantidade do escarro induzido em portadores de asma ou doença pulmonar obstrutiva crônica. J Bras Pneumol, v. 34, n. 12, p. 1026-1032, 2008. OPLUSTIL, P.C. et al. Procedimentos básicos em microbiologia. 2. ed. São Paulo: Sarvier, 2004. PAGGiARO, P. L. et al. Sputum induction. Eur Respir J, v. 20, n. 37, p. 3s-8s, 2002.

PARR, D. G. et al. Inflammation in sputum relates to progression of disease in subjects with COPD: a prospective descriptive study. Respiratory Research, v. 7, n.136, p.1-11, 2006.

PARREIRA, V. F. et al. Pressões respiratórias máximas: valores encontrados e preditos em indivíduos saudáveis. Rev. Bras. Fisioterapia, v. 11 n. 5, p. 361-368, 2007.

QUEIROZ, C.F. et al. Perfil inflamatório e imunológico em pacientes com DPOC: relação com a reversibilidade do $\mathrm{VEF}_{1 .}$ Jornal Brasileiro Pneumologia, v. 44, n. 4, p. 241-247, 2016.

RABAHI, M. F. Epidemiologia da DPOC: Enfrentando desafios. Pulmão RJ, v. 22, n. 2, p.4-8, 2013.

PEREIRA, C.A.C. Teste de função pulmonar. Sociedade Brasileira de Pneumologia e Tisiologia. p.1-12 abril, 2001.

RUFINO, R. et al. Perfil celular do escarro induzido e sangue periférico na doença pulmonar obstrutiva crônica. J Bras Pneumol. v. 33, n.5, p. 510-518, 2007.

SAKUNDARNO, M. Insufficient quality of sputum submitted for tuberculosis diagnosis and associated factors, in Klaten district, Indonesia. BMC Pulmonary Medicine, v. 9, n.16, p. 1-11, 2009.

SIPOLI, L. G. et al. Força muscular inspiratória na DPOC e sua correlação com função pulmonar e massa magra. Revista Inspirar, v. 1, n. 3, p. 84, 2009.

SOUZA, R. B. Pressões respiratórias estáticas máximas. Jornal de Pneumologia, v. 28, n. 3, p. S155-S65, 2002.

TUDER, R. M.; PETRACHE, I. Pathogenesis of chronic obstructive pulmonary disease. J Clin Invest, v.122, n.8, p. 2749-2755, 2012.

VLAHOS, R.; BOZINOVSKI, S. Recent advances in pre-clinical mouse models of COPD. Clin Sci (Lond), v. 126, n.4, p. 253-265, 2014. 
